Инга Милевич

$$
\underset{\substack{\text { Member since } \\ \text { JM14477 }}}{2019}
$$

(iD) https://orcid.org/0000-0002-4948-8655

Alberta College

Skolas 22, LV-1010, Riga, Latvia

inga.milevica@gmail.com

\title{
Стратегии перевода: средство самопрезентации автора научной публикации?
}

\section{Translation strategy: self-presentation of the author of a scientific publication?}

\section{Резюме}

Статья посвящена одному из дискуссионных терминов современного переводоведения - стратегии перевода. Типологии стратегий перевода отличаются принципами их выделения и обоснования. В статье анализируется 325 научных статей электронной библиотеки Cyberleninka, содержащих сочетание стратегия + перевод. Результаты анализа показали, что в научных статьях понятие стратегии перевода часто используется в аннотации/ключевых словах научной статьи, но в то же время никак не определяется в самом тексте статьи. В таком случае возможно предположить, что использование понятие стратегии перевода продиктовано соображениями самопрезентации автора научной статьи.

Ключевые слова: стратегия перевода, самопрезентация, научная статья, Cyberleninka.

\section{Summary}

The article is devoted to one of the controversial terms of modern translation studies, namely translation strategy. The typologies of translation strategies differ in terms of principles of their identification and justification. The article analyses the Cyberleninka electronic library and 325 scientific articles containing the combination strategy + translation. The results of the analysis showed that in scientific articles the concept of a translation strategy is often used in the abstract/keywords, but at the same time, it is not defined in any way within the article proper. In this case, it is possible to assume that the use of the concept of translation strategy is driven by considerations of self-presentation of the author of a scientific article.

Keywords: translation strategy, self-presentation, scientific article, Cyberleninka. 
Как известно, стратегия перевода относится к дискуссионным терминам современного переводоведения. На большое количество конкурирующих терминов (procedures, techniques, operations, changes, shifts, methods, replacements), несогласованность в принципах выделения и обоснования стратегий перевода указывает, напр., Ив Гамбье (Gambier, 2010, 412, 413), и находит частичное обьяснение этому:

This terminological variation can be explained partly by the disciplinary background of the researchers (comparative literature, stylistics, discourse analysis, psycholinguistics, etc.), the purpose of their investigation (pedagogical application, theoretical discussion, explanation of a problem), and/or the scope of their studies (intending to cover all possible relationships between a source text (ST) segment and a target text (TT) segment, discovering what is going on in the translator's mind, focusing on a very specific item) (Gambier, 2010, 412).

Тем не менее возможно обнаружить три основных понимания стратегии nеревода: 1. Переход между языками (Shifting between languages); 2. Решение проблемы (Solving a problem); 3. Рабочий процесс перевода (Translation working process) (Gambier, 2010, 413-415).

Однако, если обратится, в частности, к научным статьям российских исследователей, то названных оснований для понимания стратегий перевода недостаточно. Нередко причиной использования термина стратегия перевода является самопрезентация автора статьи, вследствие чего понимание стратегии перевода отходит на второй план (если вообще присутствует).

Целью данной работы явилась проверка гипотезы: если такой неоднозначный термин как стратегия перевода выносится в аннотацию/ключевые слова научной статьи, то в статье должно присутствовать его определение. В январе 2020 года в электронной библиотеке Cyberleninka для анализа были отобраны статьи, содержащие сочетание стратегия+nеревод, общее число статей - 325 (статьи не отражены в библоиграфическом списке). Cyberleninka - это самая большая российская электронная библиотека, созданная на основе парадигмы Open Science, входящая в топ-5 международного рейтинга Transparent Ranking of Repositories, а статьи включены в такие значимые базы как BAK, RSCI, ESCI, Scopus. Методы исследования - семантический, контекстуальный и статистический анализ. Для анализа использовались следующие позиции:

1. Использование сочетания стратегия перевода (варианты - переводческая стратегия, стратегия переводчика) в т. н. сильных позициях текста - аннотация и ключевые слова.

2. Присутствие или отсутствие в тексте статьи определения стратегии/я перевода.

Кроме того, учитывался жанр статьи (не принимались во внимание рецензии и обзоры); специализация автора статьи, его образование и государственная принадлежность (рассматривались статьи, написанные россий- 
скими авторами со степенью выше магистра); материал исследования (художественная литература, научная литература, аудиовизуальные тексты и др.).

Итак, понятие (от использования термин пока воздержимся) cmpameгии перевода зачастую используется без определения, в то же время именно это понятие указывается в аннотации, ключевых словах или даже названии статьи. Можно предположить, что причинами этого могут стать тенденции моды в науке, некритичность, конформизм и др., другими словами, нарушение принципов научного этоса и увлечение самопрезентацией автора научной статьи.

Самопрезентация определяется как 'any behaviors intended to convey a particular image of, or particular information about, the self to other people' (APA Dictionary of Psychology). Соответственно, самопрезентация подразумевает определенный набор моделей и приемов коммуникативного поведения, направленных на управление впечатлением - контролируемому представлению информации о себе.

Исследований самопрезентации достаточно много на материале различных средств коммуникации (особенно невербальных), гендерных ролей в коммуникации (особенно в ситуации знакомства), а также социальных сетей, публичных выступлений, корпоративных коммуникаций, детской речи, отдельных жанров (напр., биография), особенно жанров делового общения (особенно - первого рабочего интервью), однако исследований самопрезентации на материале научного дискурса немного. Среди таких исследований выделяется исследование самопрезентации в аспекте гендерных ролей (Daubman, Heatherington, Ahn, 1992), кроме того, изредка появляются исследования самопрезентации исследователя, в частности, интервьюера во время интервью (Lee, Roth, 2004). Следует упомянуть сравнительное исследование политического, судебного и научного дискурсов в аспекте самопрезентации (Аксенова, 2013). Одной из особенностей самопрезентации в научном дискурсе является косвенность.

Гораздо чаще [прямой] встречаются элементы косвенной самопрезентации, с помощью которых подчеркивается принадлежность к авторитетной научной школе, самостоятельность исследования, масштабность проделанной работы и т.п. В некоторых случаях адресант с помощью этой стратегии старается подчеркнуть свой авторитет в научном мире (Аксенова, 2013, 111).

Использование лексемы с более абстрактным значением может стать таким косвенным способом самопрезентации. Лексема стратегия является более абстрактной, и она может создать нужный автору статьи ракурс восприятия, в отличие от лексемы прием, которая является не столь абстрактной. Ср.: Стратегия 1. 'Наука о ведении войны, искусство ведения войны. Теория военной стратегии. 2. Общий план ведения войны, боевых операций. Побе- 
доносная с. 3. перен. Искусство руководства общественной, политической борьбой, а также в целом искусство планирования руководства, основанного на правильных и далеко идущих прогнозах' и прием '1. см. принять. 2. Отдельное действие, движение. Выпить стакан в два приема. 3. Способ в осуществлении чего-н. 4. Собрание приглашенных (обычно у официальных лиц) в честь кого-чего-н.' (Толковый словарь русского языка/ТСРЯ online).

Обратимся к материалу исследования, которое охватывает - 325 статей. В 11 статьях нефилологов понятие стратегии перевода было 11 раз использовано в аннотации и/или ключевых словах; в 9 статьях нет определения стратегии перевода, в двух статьях с определением производился анализ определений. Примечательно, что в статьях без определения стратегии перевода это понятие контекстуально заменяется на (сближается с такими понятиями как) тенденция, подход, выбор автора. Ср.. ...однако новые тенденции в польском стихосложении побуждают переводчика комбинировать вымеперечисленные стратегии; Следовательно, переводчик должен сделать выбор между двумя вышеуказанными подходами: переводит ли он для чтения вслух или про себя? Разумеется, одно и то же стихотворение может оказаться в обеих ситуациях. В другой статье контекстуальными заменами/сближениями становятся установка, цель: Используя математический термин, подчеркнем, что стратегия перевода является функцией установки перевода, которая, в свою очередь, является функцией цели инициатора...

Во всех 18 статьях литературоведов понятие стратегии перевода использовано в аннотации и/или ключевых словах, в 16 статьях нет определения стратегии перевода. Типичный пример контекстуальных синонимов/ сближений в статьях: B работе поднимается проблема передачи сравнений и сравнительных конструкций в художественном переводе...; и далее Вопрос о закономерностях предпринимаемых переводческих решений при передаче сравнений не универсален. Каждый переводчик находит свои стратегии и методы по преодолению трудностей перевода. При переводе сравнительных оборотов переводчик сталкивается с рядом задач: достоверно передать денотат оригинала, прагматическое значение, национальную специфику оборота; Рассмотрим специфику переводческих стратегий при передаче сравнительных конструкций с русского на осетинский язык в произведениях русской литературы; в заключении статьи: Таким образом, сравнительно-сопоставительный анализ способов передачи в художественном переводе сравнительных конструкиий на русский / осетинский язык показал основные переводческие стратегии, к которым прибегает переводчик в своей работе. Доминирующим способом передачи образов, лежсащих в основе сравнения, является калькирование - дословный перевод. В исключительных случаях переводчик прибегает к замене, опущению сравниваемых образов или описанию средствами переводящего языка передаваемого ими смысла. 
Соответственно, без определения или формулирования разницы между понятиями исчезает терминологическая точность научной статьи. Примечательно, что реализации синонимических замен контекстуального характера и семантическое сближение происходит подчас в рамках одного предложения: Выявляется специфика переводческой техники названного автора, теоретически обосновывается правомерность и плодотворность новаторского приема поэтической трансформации, делается вывод о том, что одно из несомненных достоинств книги - попытка внести в современную теорию художественного перевода новую стратегию диалога с подлинником и продемонстрировать на практике ее содержательные возможности. Далее в тексте статьи: Трансформация как концептуальная стратегия, судя по всему, коррелирует с отдельными, не совпадающими с ней видами перевода, из которых самым примечательным является свободный стих, или верлибр. И далее: По сути, выбрана продуктивная тактика перевода, отличающаяся от всех ныне сущуествующих: сонеты Шекспира, состояшие из четырнадизати строк, мастерски «уплотняются» в четыре строки авторских рубаи по персидскому каноническому образцу, а сами рубаи Хайяма «выпрялляются» в четырнадцатистрочной линейке авторских сонетов на английский манер. Еще один характерный пример: зависимость стратегии Ш. Мударриса от канонизированного в те времена маршаковского стиля оспаривать не приходится, за одним исключением; в статье далее понятие стратегии не только синонимизируется с понятием принцип, но и указывается на несоответствие выбранного, но так и не определенного инструмента исследования объекту исследования. Если подходить к подобному переводу с точки зрения историко-сочииальных условий, то можно было бы сказать так: Ш. Мударрис совместил переводческие принципы 1940-х годов с принцииами поэтического творчества 1960-х годов - холодно-рациональную ясность сталинского «века-зверя» с чувством «оттепельной» свободы. Конечно, такое определение переводческой стратегии является видимым упрощцением, вульгаризациией литературного опыта, подобно всякой редукциии, пытающейся свести сложные художественные феномены к простыл и легко прочитываемым схемам. И еще один пример: Перед нами не форенизация как стратегия сохранения при переводе маркеров иной этнокультурной идентичности. Переводческие решения Пивера и Волохонски (и частично Герни), скорее, можно обозначить термином «остранение» в том смысле, в котором его употреблял В.Б. Шкловский. Англоязычное понятие "defamiliarization», соответствующее русскоязычному «остранение», обладает бо́льиим объемом, чем «форенизация». Поэтому далее для обозначения соответствующей стратегии на всех уровнях мы будем пользоваться понятием «остранение». Тогда противоположную стратегию можно назвать «натурализацией», поскольку это понятие означает и «одомаинивание» и «придание естественности». 
Из 98 статей переводоведов 42 статьи посвящены вопросам теории перевода, в том числе, 13 - вопросам методики и 6 - видам перевода. В 14 статьях использовано определение стратегии перевода, в 5 из этих статей понятие подвергается детальному анализу; в 40 статьях понятие стратегии перевода использовано в аннотации/ключевых словах. Примечательно, что в 18 статьях используется дуальная структура: в 12 статьях - микро- и макростратегии, в одной статье - буквальный/вольный, в трех - форенизация/ доместикация, в одной - прототипоческая/адаптивная стратегия; в одной - стратегия формы и содержания; в 21 статье понятие стратегии применяется для характеристики этапов перевода, напр., стратегия понимания исходного текста и стратегия передачи. Примечательно, что 36 статей из 98 посвящены переводам художественной литературы (в 30 статьях нет определения теории перевода, в 33 статьях рассматриваемое понятие включено в аннотацию/ключевые слова; в 9 статьях (в трех использовано определение) использована дуальная структура стратегии доместикащия/ форенизация, направленная на кульутрную адаптацию/не направленная), аудиовизуальному переводу посвящено 6 статей (5 статей без определения стратегии перевода) - во всех статьях понятие стратегии перевода использовано в аннотации/ключевых словах; в двух статьях - без определения - стратегия перевода рассматривается в дуальной структуре: локализация/ стандартизация; точность/вольность; медицинскому переводу посвящены две статьи (в статьях нет определения стратегии перевода, но понятие включено в аннотацию/ключевые слова); переводу публицистики - две статьи (в обеих статьях нет определения стратегии перевода, но оно включено в аннотацию/ключевые слова); переводам туристического дискурса посвящены четыре статьи (в трех статьях нет определения статегии перевода, во всех статьях понятие включено в аннотацию/ключевые слова); переводам дискурсов политики и дипломатии посвящены четыре статьи (во всех статьях нет определения статегии перевода, но понятие включено в аннотацию/ключеные слова; в одной статье использована дуальная структура стратегии - микростратегия и макростратегия); переводу компьютерных игр посвящена одна статья (нет определения статегии перевода, но понятие включено в аннотацию/ключевые слова); индустриальному специальному переводу посвящена одна статья (нет определения статегии перевода, но понятие включено в аннотацию/ключевые слова).

Из 198 статей языковедов 124 статьи посвящены переводу художественной литературы (в том числе одна статья - переводу Библии): в 13 статьях есть определение стратегии перевода, в 115 статьях понятие включено в аннотацию/ключевые слова; 21 статья посвящена аудиовизуальному переводу (в пяти статьях есть определение, в 18 понятие использовано в аннотации/ ключевых словах); одна статья - переводу компьютерных игр (нет определения стратегии перевода, но понятие использовано в аннотации); три статьи 
посвящены переводу рекламы и публицистики (из трех статей - в одной есть определение, а в трех используется понятие в аннотации/ключевых словах; в двух использована дуальная структура стратегии перевода; доместикация/ форенизация; сохранять или изменять концептуальную составляющую исходого состава); четыре статьи - переводу экономических и финансовых текстов (из четырех статей - в одной использовано определение, во всех четырех статьях - включено в аннотацию/ключевые слова); две статьи - техническому переводу (в обеих статьях нет определения стратегии перевода, но понятие использовано в аннотации/ключевых словах, в одной - использована дуальная структура стратегии перевода - прототипическая/адаптивная); одна статья посвящена переводу дискурса туризма (нет определения стратегии перевода, но понятие использовано в аннотации; использована дуальная структура вольность/тень автора); одна статья посвящена переводам милитарного текста (есть определение стратегии перевода, понятие включено в аннотацию); три статьи посвящены переводу дискурса политики и дипломатии (во всех трех статьях нет определения стратегии перевода, но понятие использовано в аннотации; в одной статье использована дуальная структура микро - и макростратегия); семь статей посвящено переводу научных, в том числе исторических и философских текстов (в двух статьях использовано определение стратегии перевода, во всех статьях включено в аннотацию/ключевые слова; в одной статье - с определением стратегии перевода - использована дуальная структура стратегии перевода адаптивная/резистентная стратегия); одна статья посвящена переводу конференций (есть определение стратегии перевода, понятие использовано в аннотации); одна статья - переводу текстов искусства (нет определения стратегии перевода, но понятие использовано в аннотации); одна статья - переводу медицинских текстов (нет определения стратегии перевода, но понятие использовано в аннотации); семь статей посвящены переводу юридических и деловых текстов (четыре статьи с определением стратегии перевода, в каждой статье понятие использовано в аннотации), 28 статей посвящено теории и методики переводоведения, из которых шесть - теории перевода, 10 - методике перевода, 12 статей - проблемам перевода (проблемы перевода отдельных языковых единиц) - семь статей с определением стратегии перевода, в 25 определение использовано в аннотации/ключевых словах.

Предварительный анализ показал, что в статьях присутствуют и другие тенденции. Обращает на себя внимание использование контекстуальных синонимов или невнятных формулировок. Ср.: Некоторые исследователи коммерческой адаптации текстов называют такой перевод дезориентирующим [1], однако, на наш взгляд, корректней отнести его к приему стратегии замены; в статье рассматриваются стратегии перевода иноязычных вкраплений в художественном тексте на материале французского и русского языков. [...] Переводческая тактика в отношении 
латинских слов... [...]; Кроме вымеупомянутых стратегий форенизации и доместикации локализаторы видеоигр часто пропускают фрагменты текста оригинала, оставляя их без перевода (no translation strategy-cmpaтегия «без перевода») [3]. Данный прием перевода можно встретить на любом участке игры; Переводы последних лет ориентируются на стратегию сохранения художественного и эстетического воздействия текста. Переводчики (А. Адамов, А. Маркович, Ф. Морван) уделяют особое внимание передаче авторского замысла, подтекста произведения, сохранению стилистической и эстетической спеиифики подлинника. [...] Противоположная тенденция перевода связана со стремлением переводчиков акцентировать русский колорит пьес А. П. Чехова, их «славянский характер». [...] Ведущцим принципом большинства переводов второй половины $X X$ в. является «дерусификаиия» текста оригинала, отсутствие явной ссылки на Россию кониа XIX в. [...] Главная особенность переводов А. Адамова-знание им не только текста произведения, но и творчества автора в иелом. Подобные замены наблюдаются даже в рамках одного предложения: Это во многом зависит от выбранной переводчиком стратегии (способа) трансляции имени на другой язык. Отдельно стоит обратить внимание на то, что синонимы используются в заключении статей. Напр., начало статьи: Специфика перевода городской безэквивалентной лексики сводится к двум основным стратегиям: a) переводчик стремится найти в той или иной степени адекватные оригиналу номиначии, чаще всего описательные обороты, или б) вводит в текст варваризм, комментируя его в подстрочнике. В заключении статьи: Приемы и стратегии в интерпретации городского пространства в значительной мере зависят от авторских интенций.

Анализ показал, что в 242 статьях из 325 (т.е. в 74,46\% статей) не используется определение стратегии перевода, причем в 255 (т.е. в 78,46\% статей) статьях понятие включено в аннотацию/ключевые слова именно тогда, когда понятие стратегии перевода не подвергается определению. Таким образом, гипотеза не подтвердилась: не наблюдается корреляции между использованием понятия стратегии перевода в анотации/ключевых словах и введением в текст статьи определения стратегии перевода. Обращает на себя внимание факт, что часто используется дуальная структура стратегии перевода, а наиболее частым материалом исследования остается художественная литература. Эти два факта могут свидетельствовать не только о традициях и приемственности научных исследований, но и о нежелании покинуть зону комфорта исследователя-филолога. В ходе исследования также обнаружились следующие тенденции: понятие стратегии перевода в тексте статьи регулярно замещается (сближается с) понятиями приема, способа, решения, тенденции, принципа и др., что позволяет предположить, что понятие стратегии используется в целях самопрезентации автора статьи. Важно отметить, что подобное явление вокруг денотата не является спора- 
дическим явлением, а регулярным. Понятие стратегии перевода становится инструментом или маркером современного научного дискурса, видимо, потому что понятие стратегии воспринимется как более концептуальное и актуальное, чем, напр., прием, особенно в сильных позициях научной статьи - аннотациях, ключевых словах. Таким образом, утверждение об узком и широком понимании понятия стратегии перевода оказывается неполным: понятие стратегии перевода может быть использовано в целях самопрезентации автора научной статьи.

\section{БИБЛИОГРАФИЯ}

Аксенова, А.В. (2013). Стратегия самопрезентации как средство формирования имиджа в разных видах дискурса. Актуальные проблемы филологии и педагогической лингвистики, 15, 109-114.

Толковый словарь русского языка online. https://ozhegov.slovaronline.com/ (доступ: 8.12.2020).

$$
* * *
$$

Aksenova, A.V. (2013). Strategiya samoprezentatsii kak sredstvo formirovaniya imidzha v raznykh vidakh diskursa. Aktual'nye problemy filologii i pedagogicheskoi lingvistiki, 15, 109-114.

APA Dictionary of Psychology. https://dictionary.apa.org/self-presentation (dostup: 8.12.2020).

Daubman, K.A., Heatherington, L., Ahn, A. (1992). Gender and the Self-Presentation of Academic Achievement. Sex Roles, Vol. 27, Nos. 3/4, 187-204.

Gambier, Yves. (2010). Translation strategies and tactics. Handbook of Translation Studies. Volume I. Ed. Yves Gambier, Luc van Doorslaer. Amsterdam-Philadelphia: John Benjamins Publishing Company, 412-418.

Lee, Y.-J. \& Roth, W.-M. (2004). Making a Scientist: Discursive "Doing” of Identity and SelfPresentation During Research Interviews. Forum: Qualitative Social Research Social Forschung. Vol. 5, No. 1, Art. 12 January 2004.

Tolkovyi slovar' russkogo yazyka online. https://ozhegov.slovaronline.com/ (dostup: 8.12.2020). 